\title{
An Overview of Possible Aeroelastic Instabilities for Wind Turbine Blades
}

\author{
J. G. Holierhoek* \\ Unit Wind Energy, Energy research Centre of the Netherlands, P.O. Box I, I755 ZG Petten, The \\ Netherlands
}

Received November 15, 20 I 2; Revised May 3, 20 I3; Accepted May 3, 20 I3

\begin{abstract}
Aeroelastic instabilities can be disastrous for wind turbines. In the early years of wind turbines, aeroelasticity was not a problem, but once wind turbines became larger than $500 \mathrm{~kW}$, some turbines experienced problems due to aeroelastic instabilities. Therefore it is important to design wind turbines with full knowledge of possible aeroelastic instabilities. Different known possible wind turbine blade instabilities are examined and investigated. Stall flutter and flap-lag-stall flutter have been investigated in more detail, showing the possibility of these instabilities occurring on wind turbines, because these instabilities have had less exposure in wind energy literature. Negative damping of the edgewise mode, negative damping of the flap mode and classical flutter are also discussed. The examples show that the knowledge already gained in the field of helicopter aeroelasticity can be very useful. However, the differences between helicopters and wind turbines are such that the results for helicopters cannot simply be copied to wind turbines. There are several instabilities that occur on wind turbines and are less relevant for helicopters.
\end{abstract}

\section{NOTATIONS}

$\begin{array}{lll}c & \text { - Chord } & {[\mathrm{m}]} \\ c_{d} & \text { - 2D Drag coefficient: } \frac{d}{\frac{1}{2} \rho V^{2} c} & {[-]} \\ & & \\ c_{d_{\alpha}} & \frac{d c_{d}}{d_{\alpha}} & {[-]} \\ & & \\ c_{l} & -2 \text { D Lift coefficient: } \frac{l}{\frac{1}{2} \rho V^{2} c} & {[-]} \\ & & \\ & & {[-]} \\ c_{l_{\alpha}} & \frac{d c_{l}}{d_{\alpha}} & {[\mathrm{kg} / \mathrm{s}]} \\ c_{x x} & \text { - Damping in-plane } & {[\mathrm{kg} / \mathrm{s}]} \\ c_{x y} & \text { - Damping coupling } & {[\mathrm{kg} / \mathrm{s}]} \\ c_{y x} & \text { - Damping coupling } & {[\mathrm{kg} / \mathrm{s}]} \\ c_{y y} & \text { - Damping out-of-plane } & {[\mathrm{N}]} \\ D & \text { - Drag force } & {\left[\mathrm{kgm}^{2}\right]} \\ I & \text { - Mass moment of inertia } & \end{array}$

${ }^{*}$ Correspondence to: J.G. Holierhoek, Unit Wind Energy, Energy research Centre of the Netherlands, P.O. Box 1, 1755 ZG Petten, The Netherlands. E-mail: holierhoek@ecn.nl 


\begin{tabular}{|c|c|c|}
\hline$k_{\beta}$ & -Spring stiffness flap direction & {$[\mathrm{Nm} / \mathrm{rad}]$} \\
\hline$k_{\varepsilon}$ & -Spring stiffness hub & {$[\mathrm{Nm} / \mathrm{rad}]$} \\
\hline$k_{\zeta}$ & -Spring stiffness edgewise direction & {$[\mathrm{Nm} / \mathrm{rad}]$} \\
\hline$k_{\theta}$ & -Spring stiffness pitch system & {$[\mathrm{Nm} / \mathrm{rad}]$} \\
\hline$L$ & - Lift force & {$[N]$} \\
\hline$l_{x}$ & - Distance between EC and AC & {$[\mathrm{m}]$} \\
\hline$M_{\beta}$ & - Moment about flap hinge & {$[\mathrm{Nm}]$} \\
\hline$M_{\zeta}$ & - Moment about lead-lag hinge & {$[\mathrm{Nm}]$} \\
\hline$r$ & - Local radial position & {$[m]$} \\
\hline$R$ & - Blade radius & {$[m]$} \\
\hline$V$ & - Local wind velocity & {$[\mathrm{m} / \mathrm{s}]$} \\
\hline$V_{t o t}$ & - Total local velocity & {$[\mathrm{m} / \mathrm{s}]$} \\
\hline$W$ & - Total velocity & {$[\mathrm{m} / \mathrm{s}]$} \\
\hline$\alpha$ & - Angle of attack & {$[\mathrm{rad}]$} \\
\hline$\beta$ & - Flapping angle & {$[\mathrm{rad}]$} \\
\hline$\tilde{\beta}$ & $\begin{array}{l}\text { - Flapping angle including contribution from hub } \\
\qquad \rho c_{l} c R^{4}\end{array}$ & {$[\mathrm{rad}]$} \\
\hline$\gamma$ & - Lock number $\gamma=\frac{\rho_{\alpha}}{I}$ & {$[-]$} \\
\hline$\varepsilon$ & - Hub degree of freedom angle & {$[\mathrm{rad}]$} \\
\hline$\zeta$ & - Lead-lag angle & {$[\mathrm{rad}]$} \\
\hline$\widetilde{\zeta}$ & - Lead-lag angle including contribution from hub & {$[\mathrm{rad}]$} \\
\hline$\theta$ & - Pitch angle & {$[\mathrm{rad}]$} \\
\hline$\theta_{0}$ & - Offset for the angle of attack relative to pitch angle & {$[\mathrm{rad}]$} \\
\hline$\nu_{\beta}$ & - Non-dimensionalised rotating flap frequency & {$[-]$} \\
\hline$\nu_{\varepsilon}$ & - Non-dimensionalised hub frequency & {$[-]$} \\
\hline$\nu_{\zeta}$ & - Non-dimensionalised rotating lead-lag frequency & {$[-]$} \\
\hline$\rho$ & - Air density & {$\left[\mathrm{kg} / \mathrm{m}^{3}\right]$} \\
\hline$\omega_{\beta}$ & - Flap frequency & {$[\mathrm{Hz}]$} \\
\hline$\Omega$ & - Rotational velocity & {$[\mathrm{rad} / \mathrm{s}]$} \\
\hline \multicolumn{3}{|c|}{ SUBSCRIPTS } \\
\hline 0 & -Equilibrium state & \\
\hline \multicolumn{3}{|c|}{ SUPERSCRIPTS } \\
\hline$B$ & - Direction of eigenmodes & \\
\hline $\begin{array}{l}R \\
()^{\prime}\end{array}$ & $\begin{array}{l}\text { - Direction of in-plane - out-of-plane } \\
-\frac{\partial}{\partial}\end{array}$ & \\
\hline
\end{tabular}

\section{INTRODUCTION}

Over the last decades the size of wind turbines has rapidly increased. Though there were no aeroelastic problems for the first wind turbines, since the rated power has become higher than $500 \mathrm{~kW}$ aeroelasticity has become relevant as some turbines suffered from instabilities. To be able to prevent instabilities, knowledge of basic aeroelasticity and aeroelasticity of rotors is a necessity. Note that one has to distinguish between aeroelastic instabilities and resonance. In the case of aeroelastic instabilities, one of the modes becomes negatively damped. While resonance occurs when a forcing frequency is too close to one of the natural frequencies, see e.g. $[1,2]$. In the remainder of this article the focus will be on the possible aeroelastic blade instabilities, not on resonance.

Helicopter aeroelasticity has been an important field of research from the beginning of helicopter development, while for wind turbines initially aeroelasticity was not important. 
Only once some experimental wind turbines such as the Dutch KEWT $[3,4]$ showed problems, it became a relevant research activity. And once the size of commercial wind turbines increased towards $40 \mathrm{~m}$ in diameter and a rated power of around $500 \mathrm{~kW}$, aeroelastic problems were for the first time encountered on commercial wind turbines by some manufacturers $[5,6,7]$. There are however many similarities between the fields of wind turbine aeroelasticity and helicopter aeroelasticity $[8,9]$. Therefore it can be very fruitful to use the elaborate existing literature concerning helicopters and investigate if similar instabilities could occur on wind turbines and if so, under which circumstances. Of course there are also relevant differences between wind turbines and helicopters, therefore there will be instabilities that can occur on wind turbines and not on helicopters and vice versa.

There are many publications addressing one or more possible aeroelastic instabilities for wind turbines, while in this article an overview is given of all possible blade instabilities that are reported in literature. For example Eggleston and Stoddard [10] give a limited overview of some of the possible blade instabilities, also based on the knowledge available from helicopter research. In [11] a literature survey is given on possible aeromechanical instabilities for helicopters and wind turbines, unfortunately some of the known wind turbine instabilities were not included in that survey, e.g. negative damping of the edgewise mode while this was the reason that some blades suffered damage $[5,6]$. In [12] Hansen gives a very clear description of the most relevant possible instabilities, but not all known possible blade instabilities are included. In [13] next to an extensive overview of the state of the art in wind turbine aeroelasticity, a short overview is given of some possible wind turbine instabilities.

In the present article a complete overview is given of the currently known or expected possible instabilities for wind turbine blades. Attention is given to instabilities that have not yet been elaborately discussed in wind turbine literature, with, when relevant, a short discussion on the equivalent helicopter instability.

An overview of possible blade instabilities of wind turbines, including a discussion on the aeroelastic differences between a helicopter and a wind turbine, is given in the next section. This is followed by a section that shortly discusses the shortcomings of isolated blade analysis when compared to complete turbine analysis and the effect that the other components of a wind turbine can have on the stability of the blades. Finally the conclusions are given.

\section{BLADE INSTABILITIES}

In this section different possible blade instabilities are discussed. First the differences between helicopters and wind turbines, from an aeroelastic point of view, are described. Then the single mode instabilities are addressed in sections 2.2 and 2.3. All three of these instabilities are stall induced instabilities. The other blade instabilities entail two or three coupled modes. These are discussed in the last two sections. One should however note that for all these blade instabilities, the presence of other structural components when looking at a complete wind turbine, can have a significant effect. The directions of the vibration of an eigen-mode can be influenced by the presence of the other components of the wind turbine structure. The frequencies are also influenced by the other components. These effects must always be taken into account when designing a wind turbine as discussed in section 3 .

\section{I. Comparing wind turbines to helicopters}

When looking at known instabilities from helicopter aeroelasticity and the possible risk for wind turbines, it is important to be aware of the similarities as well as of the differences between helicopters and wind turbines from an aeroelastic point of view. Similarities between helicopters and wind turbines include that they both have rotating blades that are lift 
generating devices and rotors which are far from rigid. One important difference between helicopters and wind turbines is the direction of the aerodynamic forces and the angles of attack during operation as discussed below.

For most wind turbine designs, the rotor shows more similarities to a hingeless helicopter rotor than to an articulated rotor. In contrast with the articulated helicopter rotor, the hingeless rotor does not always have dampers in the lead-lag direction. This makes the field of aeroelasticity more important, as it becomes possible for the usually low damped lead-lag modes to become unstable. An important difference however, concerns the non-dimensional rotating flap- and edgewise frequencies which are much higher for wind turbines than those for hingeless helicopters.

Other dissimilarities between wind turbines and helicopters are the larger planform area of wind turbines and the larger twist of the blades. For a wind turbine the chordwise location of the blade mass axis is usually behind the aerodynamic centre which also differs from helicopters. Another difference during operation is the torque of the wind turbine that continually changes. On top of that, for variable speed turbines the rotor speed is also not constant in contrast with helicopters [10].

The conditions in the flow around a wind turbine at zero yaw are rather similar to the flow conditions for a helicopter in descending vertical flight. There are of course a few important differences in the flow conditions. The most fundamental difference is the direction of the resulting force aligned with the plane of rotation. On a wind turbine the aerodynamic forces result in a torque that causes the rotation of the blade, while on a helicopter the torque must be provided by an engine to overcome the aerodynamic forces in the plane of rotation. Another important distinction is the difference in the angle of attack during operation. A wind turbine will often operate in or near stall, while a helicopter should not operate in stall. The

Lock number $\left(\gamma=\frac{\rho c_{c} c R^{4}}{I}\right.$, representing the ratio of aerodynamic forces to inertial forces on a blade) also differs, for wind turbines it is significantly lower than for helicopters.

\subsection{Stall induced vibrations in edgewise and flapwise direction}

Once the size of wind turbines increased to approximately $40 \mathrm{~m}$ in diameter, for the first time aeroelastic problems were encountered on some commercial wind turbines. In 1994 Stiesdal [5] reported problems with edgewise vibrations on a stall regulated wind turbine with a 37 meter rotor diameter. This problem had not been evident on earlier wind turbines, but with the increase in size it suddenly became an important issue for wind turbines. Damage and even loss of blades was reported by Møller in 1997[6]. Another example of a blade that suffered from this instability was the Aerpac APX40T blade[7]. These blades were mounted on $600 \mathrm{~kW}$ turbines with a 43 meter rotor diameter. They showed a severe instability at high winds. As described by Anderson et al. [7], this problem was solved by Aerpac by installing a mechanical damper inside the blades.

The vibrations of the APX40T blades were caused by negative damping of the first edgewise mode. This resulted in divergent oscillations of the blades at their first edgewise eigenfrequency during high wind operation. The problem was aggravated by the fact that the phase difference between the three APX40T blades in this vibration was such that there was a zero net oscillatory torque on the rotor shaft. Therefore it was not possible for the power train to suppress this instability [7]. This problem was not a case of resonance, it was due to the negative damping of the first edgewise eigenmode. The frequency of the first edgewise mode was less relevant, it was the shape of the eigenmode which had the strongest influence on 
the stability of the mode. Some out-of-plane motion in the first edgewise mode would have had a stabilising effect on the stability of this mode. Because the mode shape is determined by the stiffness and mass distribution, these distributions have significant effect on the instability [14].

The reason for this instability can be illustrated using the equations of a simplified model. Petersen et al. [14] have derived a simple model that gives insight in the aerodynamic damping of the first flapwise and first edgewise blade mode. The model is illustrated in figure 1. By determining the aerodynamic forces acting on this aerofoil in the $X_{R}$ and $Y_{R}$ direction, assuming small angle of attack variations, it is possible to determine damping coefficients in these directions for this model [14,15]:

$$
\begin{aligned}
& c_{x x}^{R}(r \Omega, V)=-\frac{\partial F_{x}^{R}}{\partial(r \Omega)}=\frac{1}{2} c \rho \frac{r \Omega}{W}\left[\left(\frac{2 r^{2} \Omega^{2}+V^{2}}{r \Omega}\right) C_{d}-V C_{d_{\alpha}}-V C_{l}+\frac{V^{2}}{r \Omega} C_{l_{\alpha}}\right] \\
& c_{x y}^{R}(r \Omega, V)=\frac{\partial F_{x}^{R}}{\partial V}=\frac{1}{2} c \rho \frac{r \Omega}{W}\left[-V C_{d}-r \Omega C_{d_{\alpha}}+\left(\frac{2 V^{2}+r^{2} \Omega^{2}}{r \Omega}\right) C_{l}+V C_{l_{\alpha}}\right] \\
& c_{y x}^{R}(r \Omega, V)=-\frac{\partial F_{y}^{R}}{\partial(r \Omega)}=\frac{1}{2} c \rho \frac{r \Omega}{W}\left[-V C_{d}+\frac{V^{2}}{r \Omega} C_{d_{\alpha}}-\left(\frac{2 r^{2} \Omega^{2}+V^{2}}{r \Omega}\right) C_{l}+V C_{l_{\alpha}}\right] \\
& c_{y y}^{R}(r \Omega, V)=\frac{\partial F_{y}^{R}}{\partial V}=\frac{1}{2} c \rho \frac{r \Omega}{W}\left[\left(\frac{2 V^{2}+r^{2} \Omega^{2}}{r \Omega}\right) C_{d}+V C_{d_{\alpha}}+V C_{l}+r \Omega C_{l_{\alpha}}\right]
\end{aligned}
$$

In these equations $c_{x x}^{R}$ is the aerodynamic damping for a deformation in the direction of the rotor plane. The edgewise eigenmode of a wind turbine blade will not be purely in-plane, it will include some out-of-plane motion as well, therefore the damping coefficients in equation 1 should be transformed to the directions that coincide with the directions of the vibration in the eigenmode. This gives for the damping in edgewise $c_{x x}^{B}$ and flapwise direction $c_{y y}^{B}[14]$ :

$$
\begin{aligned}
& c_{x x}^{B}=\cos ^{2} \theta_{R B} c_{x x}^{R}+\sin ^{2} \theta_{R B} c_{y y}^{R}+\sin \theta_{R B} \cos \theta_{R B}\left(c_{x y}^{R}+c_{y x}^{R}\right) \\
& c_{y y}^{B}=\sin ^{2} \theta_{R B} C_{x x}^{R}+\cos ^{2} \theta_{R B} c_{y y}^{R}-\sin \theta_{R B} \cos \theta_{R B}\left(c_{x y}^{R}+c_{y x}^{R}\right)
\end{aligned}
$$

with $\theta_{R B}$ the angle defining the direction of the vibration, the so-called structural pitch angle.

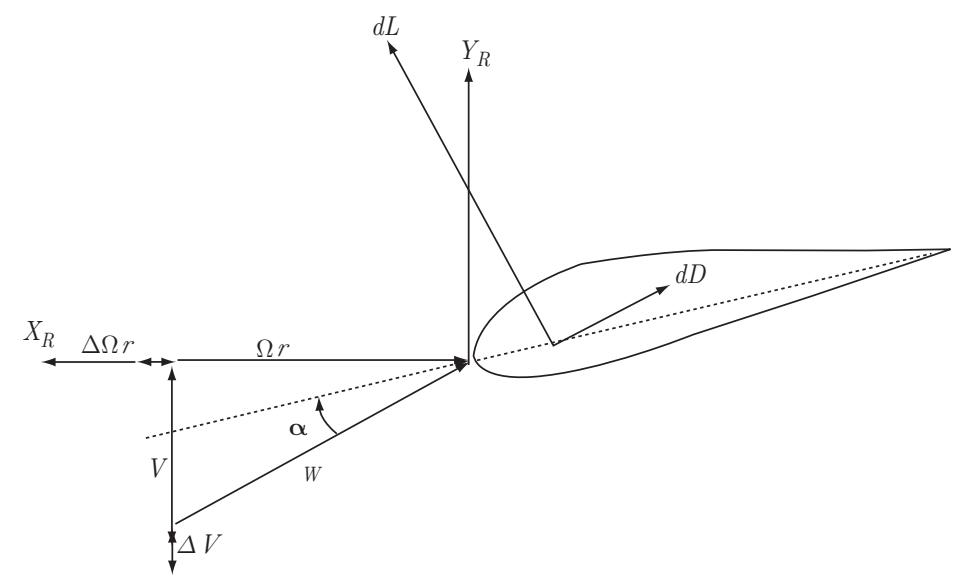

Figure 1: The aerodynamic forces acting on an element due to the local wind velocity. By looking at changes of the wind speed and rotational speed of the rotor, corresponding damping matrix can be derived. 
From equation 1 it can be seen that $c_{x x}^{R}$ is often negative, while $c_{y y}^{R}$ is usually positive. Therefore it necessary to have some out-of-plane motion in the edgewise mode, as this will improve the damping of the edgewise mode compared to a mode that is purely in-plane.

Using equations 1 and 2 , it is possible to determine the damping that comes from linearised steady aerodynamics for a wind turbine blade element as a function of the direction of the vibration. As an example the values for $c_{x x}^{B}$ are shown in figure 2 for $-90^{\circ}<\theta_{R B}<90^{\circ}$. These values were calculated for a fictive active stall regulated turbine design with an $80 \mathrm{~m}$ diameter [16] at an undisturbed wind speed of $16 \mathrm{~m} / \mathrm{s}$. This wind speed resulted in a local wind velocity ( $V$ in equation 1) of $14.4 \mathrm{~m} / \mathrm{s}$ which is assumed constant for the entire rotor plane. The damping values were calculated for four different radial positions $[17,18]$. The data used for these calculations are given in table 1.

Typical values for the structural pitch angle for wind turbines are between -20 and 0 degrees. For the example shown in figure 2, the aerodynamic damping of the edgewise mode is negative for all four illustrated radial distances in this structural pitch range.

The blade flapwise instability is another single mode instability. It is possible for the damping of the flap mode, represented by $c_{y y}^{B}$, to become negative. When the blade is stalled,

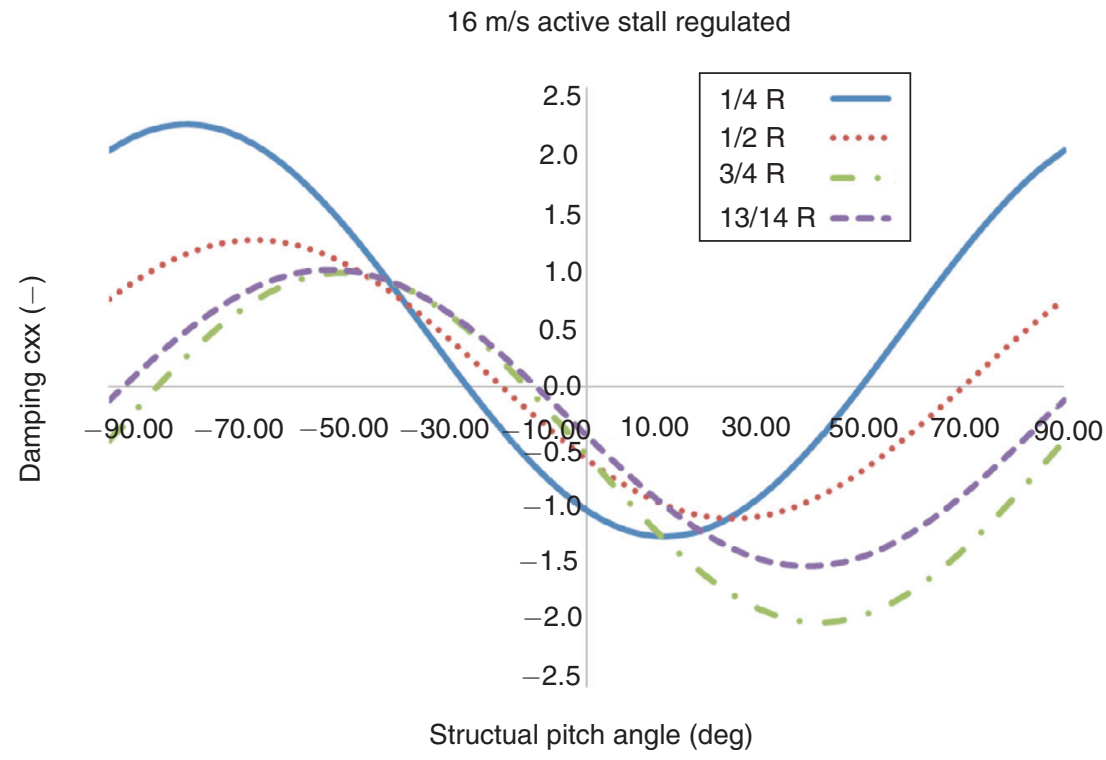

Figure 2: Non dimensional values for the damping $\frac{c_{x x}^{B}}{0.5 \rho c r \Omega}$ as functions of the structural pitch angle

for $16 \mathrm{~m} / \mathrm{s}$ at four different radial distances along the blade. The pitch setting of a stall regulated turbine has been used.

Table I: Values at the different cross sections used for the calculation of the aerodynamic damping values

\begin{tabular}{lccccc}
\hline & & $\mathbf{1 / 4} \mathbf{R}$ & $\mathbf{1 / 2} \mathbf{R}$ & $\mathbf{3 / 4} \mathbf{R}$ & $\mathbf{1 3 / 1 4} \mathbf{~ R}$ \\
\hline$t / c$ & {$[-]$} & 0.5 & 0.24 & 0.19 & 0.18 \\
$\alpha$ & {$[d e g]$} & 42 & 28 & 24 & 22 \\
$c_{l}$ & {$[-]$} & 1.04 & 1.15 & 1.19 & 1.21 \\
$c_{d}$ & {$[-]$} & 0.59 & 0.27 & 0.29 & 0.23 \\
$c l_{\alpha}$ & {$[-]$} & -0.75 & -0.13 & -0.57 & -0.57 \\
$c d_{\alpha}$ & {$[-]$} & 1.36 & 1.39 & 1.79 & 1.79 \\
$r \Omega$ & {$[\mathrm{m} / s]$} & 16.7 & 34.8 & 52.8 & 67.3 \\
\hline
\end{tabular}


the slope of the lift curve $C_{l_{\alpha}}$ can become negative, changing the stabilising effect to a destabilising effect. If $C_{l_{\alpha}}$ has a negative value, a flap motion will not be counteracted by the change in force caused by the change of the angle of attack, the change in force will be in the same direction as the flap motion.

The flapwise instability can be a realistic problem for stall regulated wind turbines. Over thirty years ago Lundsager [19] already reported increased loads and severe vibrations in flapwise direction for the Nibe turbines, when they were operating in stall. The Nibe A and Nibe B turbines both had a diameter of 40 meters.

A few years later, measurements conducted by ECN on their two-bladed $25 \mathrm{~m}$ test turbine with the 20-WPX-THR blades confirmed the possibility of a flapwise instability when the blade is operating in stall [20]. The instability was also in-fluenced by the fact that the flapping frequency was very close to $3 \mathrm{P}$ as well as close to the drive train natural frequency, which further deteriorated the instability [20]. Note however that it was not a case of resonance, the main reason for the instability was the negative damping of the flapwise mode.

Wind turbines often operate in stall, especially active and passive stall regulated wind turbines. Therefore the possibility of the flapwise instability is relevant for wind turbines, especially when taking into account that the flapwise mode will also include some badly damped in-plane motion. The damping of the flapwise mode is also illustrated in figure 2: the damping for the flap mode is equal to the value for $c_{x x}^{B}$ at $\theta_{R B}+90^{\circ}$. Therefore, for typical structural pitch angles the damping in the flap direction corresponds to the values of $c_{x x}^{B}$ for $\theta_{R B}$ in the range of $70^{\circ}$ to $90^{\circ}$. So for the given example in the graph, the damping of the flapwise motion is positive for the two inboard stations, but for the two outboard stations it is negative.

Using detailed models with nonlinear unsteady aerodynamics, it has been shown by Petersen et al. in [14] that the stall induced negative damping of the first flapwise mode will not occur on small or medium sized stall regulated wind turbines, but large (around $40 \mathrm{~m}$ in diameter or more) stall regulated wind turbines can suffer from this instability, especially when the structural pitch angle is large.

What becomes very clear from the above discussion is that the direction of the vibration of each mode is of great importance [12].

In helicopters the badly damped lead-lag or edgewise mode is often damped using some type of artificial damper. Retreating blade stall is a known problem for helicopters which concerns the negative damping of the flapwise mode for the retreating blade. This results in significant vibrations and the helicopter will roll over. This possible instability limits the speed at which helicopters are able to fly.

Another instability that is known for helicopters and that could be seen as a special case of a stall induced vibration concerning negative damping of the edgewise mode is the pitch-lag instability. In articulated rotors this intability is caused by the pitch control linkage or the inclination of the lag hinge axis whereby an increase in lag angle results in a decrease in pitch angle [21]. (Note that for helicopters the pitch angle is defined such that a decrease in pitch angle reduces the angle of attack.)

There is a relatively simple physical explanation for the instability. Assume the coupling to be such that an increase in the lead-lag angle will result in a decrease of the pitch angle. If the blade is operating in the linear part of the lift curve, this will result in a decrease of the lift force. The smaller lift force will result in a smaller average flapping angle, which in turn gives rise to a Coriolis moment: $2 \beta_{0} \dot{\beta} \Omega I$. As the flap frequency is significantly below the lead-lag frequency in the case of an articulated rotor, the blade will flap at approximately the lead-lag frequency. Therefore the flapping velocity $\dot{\beta}$ can be approximated as a linear function of the lead-lag velocity $\dot{\zeta}$. This means that the Coriolis moment can be considered as a viscous damping 
moment and when the coupling between lead-lag and pitch is such that an increase in the lead-lag angle results in a decrease in the pitch angle, the Coriolis moment provides a negative contribution to the damping. According to Bramwell [22], the instability is only possible for articulated helicopter blades when the blade pitch increases when the blade moves forward in the lead-lag motion. In general the instability will result in a limit cycle oscillation.

For hingeless helicopter blades the coupling between pitch and lead-lag angle is due to structural coupling. The instability is usually prevented in hingeless helicopter blades by designing the blade according to matched stiffness, whereby the induced pitch moment due to flapping is cancelled by the induced pitch moment due to the lead-lag motion [10].

For wind turbine blades the coupling between pitch and lead-lag angle is, similar to the hingeless rotor blades, due to the structural coupling. However, the flexibility in the pitch system can also play a role.

The structural coupling results in a change of the blade chord direction when the blade is bent in lead-lag direction. This is for example the case when a blade has a strong flapwise deformation, due to loads or due to a pre-bent. In case of a large flapwise deformation, a change in lead-lag direction gives rise to a change in the chord direction. Note that in this instability, the 'pitch' is not actually a degree of freedom, it is coupled to the lead-lag deformation. Also the flap degree of freedom (not the steady deformation resulting in the coupling) is needed in the derivation of the governing equations of motion, but as it will follow the frequency of the forcing function, its role is somewhat limited in the instability. It actually concerns a first lead-lag mode with some torsional component (and of course some flapwise component) that becomes negatively damped.

In [15] the influence of pitch coupling on the aerodynamic damping is analysed, showing that in some cases the coupling can result in an increase of the damping, but it can also reduce the damping of the mode, depending on the phase difference between the edgewise or flapwise bending and the pitch (in phase vs. counter phase), but also depending on the angle of attack (operating in stalled condition or not) and on the structural pitch angle. An in phase or in counter-phase pitch variation with the edgewise or flapwise oscillation, could represent a mechanical coupling between flap and pitch or edge and pitch. However, for larger blades, the torsional stiffness becomes more relevant and vibration modes might show up, where there is torsional deformation in the edgewise mode, which could be in any phase relation with the bending deformation.

During the UpWind project the design of very large wind turbines (8-10 MW) was investigated. One activity concerned the effect of the coupling between bending and torsion due to large deformations [23]. In [24] an example is shown that illustrates that there is indeed significant coupling between lead-lag and torsional deformation in large wind turbine blades due to the flapwise deformation. The relevance of including the geometric nonlinearities is shown, as the damping of the first edgewise mode is strongly influenced by these nonlinear effects. The damping of this mode is reduced whereby the stall induced vibrations become a possible instability, if the blade is in stall. The torsional deformation present in the lead-lag modes did not result in an instability in the attached flow regime, however in [15] it was shown that the influence of torsional deformations on the damping of the edgewise mode can be negative in the attached flow regime. Therefore the mechanism behind the pitch-lag instability for hingeless helicopter rotors can result in an instability for a wind turbine, when the deformations in flapwise directions become large resulting in significant coupling between the edgewise and torsional deformations. This instability can be regarded as a special case of negative damping of the edgewise mode. 


\subsection{Stall flutter}

The classical form of stall flutter, a third stall induced vibration, also involves a single mode, this time it is the first torsional mode. The mechanism behind this instability can easily be explained. Assume a gust that increases the angle of attack while the blade was already in stall. The forces will then decrease with an increasing angle of attack. If the aerodynamic centre is in front of the centre of twist, the smaller forces result in a decrease of the torsional deformation. This in turn reduces the angle of attack. For a smaller angle of attack in stall the force will again increase resulting in a cycle that can become unstable. In helicopters this instability can occur during combinations of high thrust and/or high forward speed $[25,26]$. In high speed forward flight a blade can enter and exit the stalled condition during each rotation.

The instability is not likely to occur during normal operating conditions in wind turbines due to their much higher stiffness in torsion. However, when there is a flexible pitch control system or a pitch link failure it is possible to occur according to Eggleston and Stoddard [10].

The pitch control system of the FLEXTEETER, a test turbine of ECN, was an example of a flexible pitch control system that resulted in stall flutter [27]. The FLEXTEETER turbine was a turbine with a diameter of $25 \mathrm{~m}$. with a flexbeam and an elastomeric teeter rotor combined with passive tip control [28]. The tip part of the blade would undergo a rotation (pitching) due to the centrifugal forces if the rpm would be above a certain maximum value. The tip control was used in combination with a variable speed conversion system as a power control device. During start-up at high wind speeds $(17 \mathrm{~m} / \mathrm{s})$ and during free run operations at around the same wind speed, instabilities were observed. There were large torsional deflections of the control tips combined with large excitations of the second flap mode. The blade and tip were operating in stall during the high wind speed start ups. As the flap motion was in phase with the torsional deformation, it was concluded that the instability was a case of classical stall flutter. The instability was solved by stiffening the torsional connection of the tip and adding a damper [27]. In practice the stall flutter instability will result in a limit cycle oscillation [29].

To show an example of this instability, a 2-D model can be used, with only pitching/torsion as a degree of freedom, as illustrated in figure 3 . The location of the elastic centre (EC) is behind the aerodynamic centre (AC) at a distance $l_{x}$ as shown in the figure. A torsional spring located in the elastic centre counteracts the aerodynamic forces,

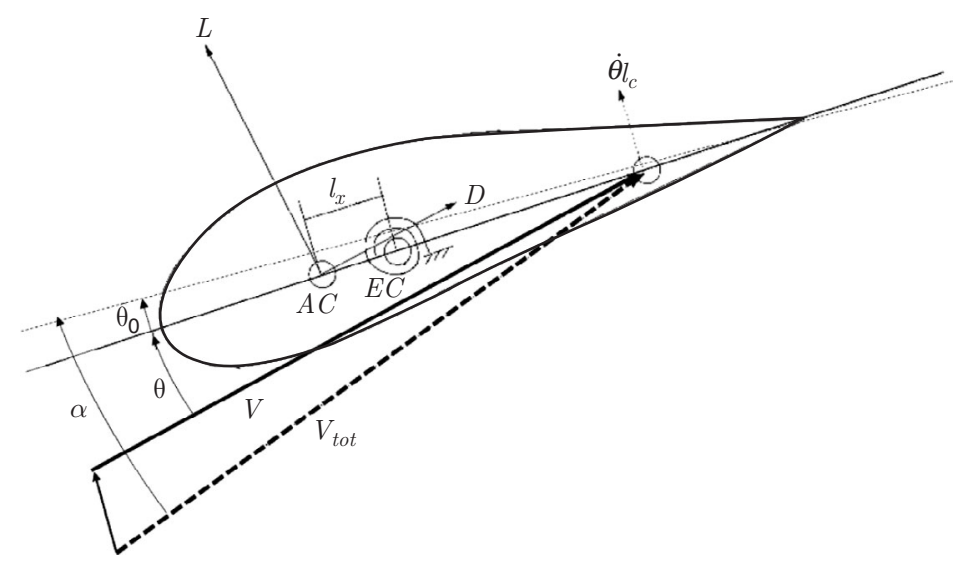

Figure 3: A pitching aerofoil model. 
modelling the torsional stiffness of the blade. The local velocity is calculated at the collocation point in the three-quarter chord point [30]. The velocity consists of a constant $V$ at an angle $\alpha$ plus the contribution due to the torsional motion of the blade. If the aerodynamic centre is in the quarter-chord point, this velocity component becomes $\dot{\theta}\left(\frac{1}{2} c-l_{x}\right)$. The blade is set at such a pitch angle that the neutral position for the torsional spring is at $\theta=0.15 \mathrm{rad}$. For the angle of attack an offset $\theta_{0}$ relative to the angle $\theta$ is assumed.

The governing equation can be derived to be:

$$
\ddot{\theta}=\frac{-k_{\theta}(\theta-0.15)+V_{t o t}^{2} c \rho l_{x}\left(c_{l} \cos \theta+c_{d} \sin \theta\right)}{I_{\theta}}
$$

with $V_{\text {tot }}^{2}=(V \cos \theta)^{2}+\left(V \sin \theta+\dot{\theta}\left(\frac{1}{2} c-l_{x}\right)\right)^{2}$ and the angle of attack that is used to determine the lift- and drag coefficient is: $\alpha=\arctan \left(\frac{V \sin \theta+\dot{\theta}\left(\frac{1}{2} c-l_{x}\right)}{V \cos \theta}\right)+\theta_{0}$.

These equations can be used in a time simulation. The lift- and drag-curves for the NACA 63418 aerofoil have been used to calculate the aerodynamic forces. Note that the effect of dynamic stall is not included in this analysis. This is because it is only meant as an illustrative example, not as a model that will be used to determine the actual stability of a wind turbine. The effect of dynamic stall is expected to be significant [31] just as it is in the pitch-flap instability (classical flutter).

The results of the simulations, using the values shown in table 2 , are illustrated in figure 4. This figure shows the results of a simulation for a realistic torsional stiffness of a large wind turbine blade on the left. Even though the operation is in stall, which is visible in the lower graph showing the attained angles of attack, the vibration is damped. The plot on the right hand side shows a simulation where the stiffness is suddenly decreased after 1.5 seconds, simulating a fault in the pitch system. Of course this results in a significantly larger torsional angle for the equilibrium position, but it can also be seen that the vibration becomes unstable. The effective lift coefficient range attained during this simulation is also shown in the bottom figure.

Table 2: Input values

\begin{tabular}{lc}
\hline Variable & Value \\
\hline$k_{\theta}$ & $22.8 \mathrm{E} 4 \mathrm{Nm} / \mathrm{rad}$ \\
$k_{\theta}$ - with pitch fault & $1.14 \mathrm{E} 4 \mathrm{Nm} / \mathrm{rad}$ \\
$c$ & $1.8 \mathrm{~m}$ \\
$\theta_{0}$ & $6^{\circ}$ \\
$V$ & $57.0 \mathrm{~m} / \mathrm{s}$ \\
$I_{\theta}$ & $80 . \mathrm{kgm}^{2}$ \\
$l_{x}$ & $0.2 \mathrm{~m}$ \\
$\rho$ & 1.225 \\
\hline
\end{tabular}



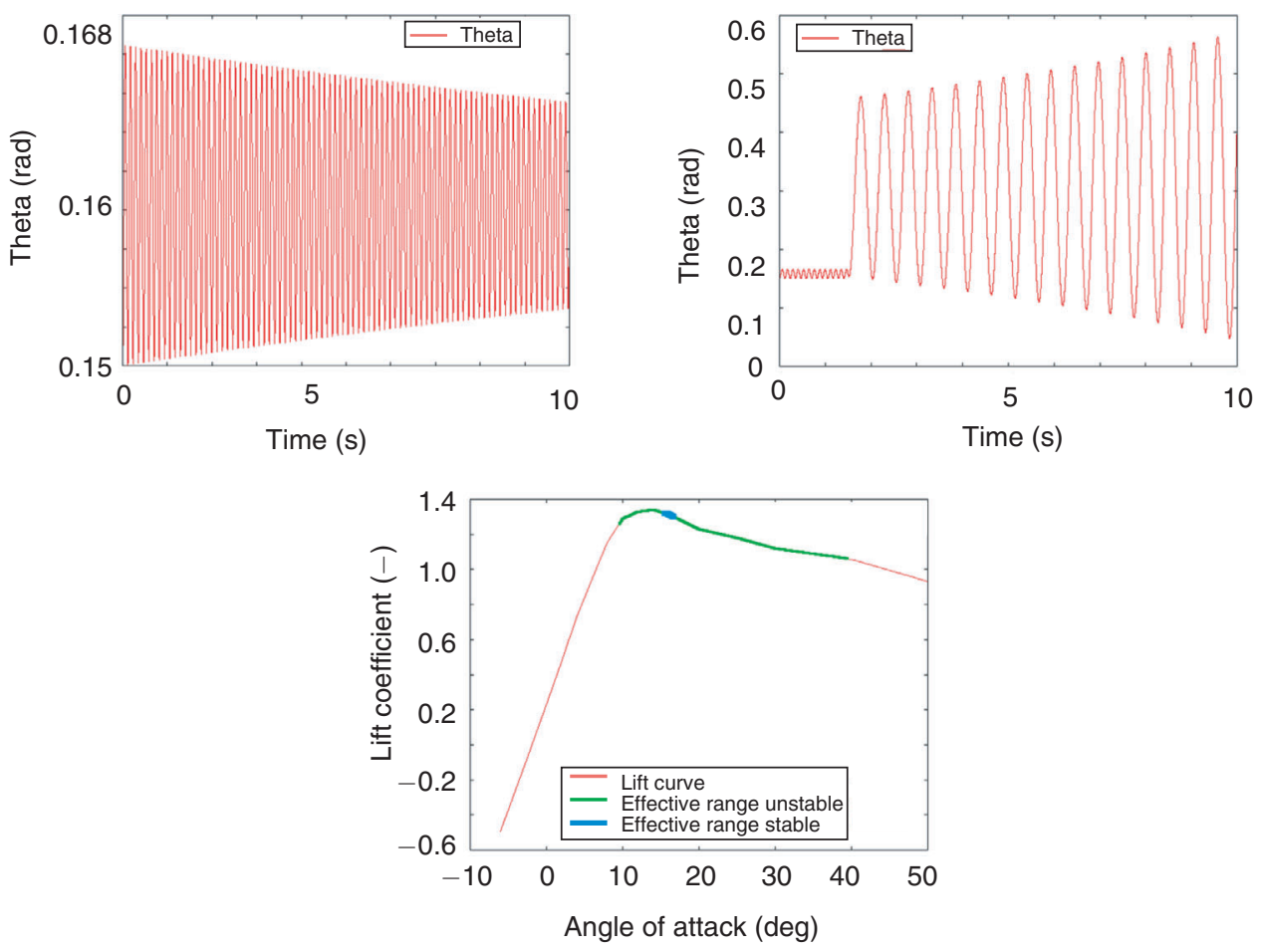

Figure 4: The pitch angle $\theta$ for a pitching aerofoil model resulting in damped vibrations for a normal stiffness (left), but a sudden reduction in stiffness results in an unstable motion of the aerofoil (right). For both cases the effective lift coefficient range attained during the simulations against the angle of attack are shown in the bottom figure.

\subsection{Flap-lag flutter, including flap-lag-stall flutter}

Flap-lag flutter is a known blade instability for hingeless and articulated helicopter rotors [32, 33]. It has been observed on some hingeless rotor blades when operating at high thrust or high pitch levels. It is an unstable oscillation that is caused by the coupling of the flap and lead-lag motions. It is a mild instability when compared to e.g. classical blade flutter (see section 2.5), but if nothing is done to prevent the instability to continue for several cycles, it can become destructive, the increasing amplitude can result in structural failure after several cycles. As it is a mild instability adding a small damper can be sufficient to prevent problems.

The flap-lag instability is caused by the coupling of two changing moments. First, there is the in-plane Coriolis moment due to the flapping velocity. E.g. for a simple isolated blade model with a moment of inertia about flap- and lead-lag hinge $I$, rotational velocity $\Omega$, flapping DOF $\beta$, this will be: $-2 I \Omega \beta_{0} \dot{\beta}$. Second, there is the alteration of the flapping centrifugal moment due to the change of the in-plane velocity by the lead-lag velocity $(\dot{\zeta}): 2 I \beta_{0} \dot{\zeta}$. However, the aerodynamic forces also couple, a change in lead-lag velocity will have an effect on the flapwise moment and vice versa.

Ormiston and Hodges [33] derived basic flap-lag equations for a centrally hinged rigid blade with spring restraints. In this derivation a linear lift coefficient was assumed and no precone, but the steady flap angle was taken into account. From these equations it was concluded that, if there was no elastic coupling, the instability can only occur when $\Omega<\omega_{\beta}<\sqrt{2} \Omega$ For helicopters this range holds realistic values for the blade flapping frequency. For wind turbines, the flap frequency will be much higher than this (e.g. approximately $3.5 \Omega$ for a diameter of $80 \mathrm{~m}$ ). The operating conditions for a wind turbine are however very different 
from a helicopter, so maybe the range found by Ormiston and Hodges is not conclusive for wind turbines. For example, helicopters rarely operate near or in the stall region, the onset of stall is a hard boundary for helicopters that severely limits their performance, while wind turbines often operate with a considerable amount of flow separation and blade stall [34], especially the stall regulated and active stall regulated turbines. More importantly, the equations that resulted in the limited range of possible instability frequencies were very basic, Ormiston and Hodges themselves showed that for example a pre-cone angle can have the effect that the limits on the flap frequency no longer apply [33]. The coupling between flap and lead-lag motions also has a significant effect [33]. Ormiston and Bousman showed that operating near stall is another case that changes the stability boundaries significantly [35].

When looking at the literature available about this instability for wind turbines, there are very few sources that give some information about the possibility of this instability to occur on wind turbines. Eggleston and Stoddard [10] provide a short description concerning this instability. However, that description and the conclusions are purely based on the basic results from Ormiston and Hodges [33], while many assumptions were used in the derivation, that will not apply to wind turbines. Therefore the conclusions from [10] do not suffice for wind turbines.

During the Dutch STABTOOL projects [36,37], it was concluded that this instability could be the cause of problems on large stall regulated wind turbines [38].

As described in the introduction, large wind turbines have shown some unexpected instabilities. Because there were no stability problems on smaller turbines it was assumed in the STABTOOL projects that these instabilities were scale dependent. Investigating the natural frequencies of several blades of different sizes showed that the manufacturers scaled their turbines up in such a way that the difference between the flap frequency and the lag frequency became smaller $[17,36,39]$. The lead-lag frequency scaled by approximately $\frac{1}{R}$, but the flap frequency scaled by approximately $\frac{1}{R^{0.8}}$.

To investigate the possibility of flap-lag flutter, a simple isolated blade model was created and analysed during the STABTOOL projects. In [38] the model of an isolated blade with central hinges with a distinct hinge order: hub $(\varepsilon)$, lead-lag $(\zeta)$ and flap $(\beta)$ is described, with a constant pitch setting between the hub angle and the lead-lag angle, see figure 5 . This model

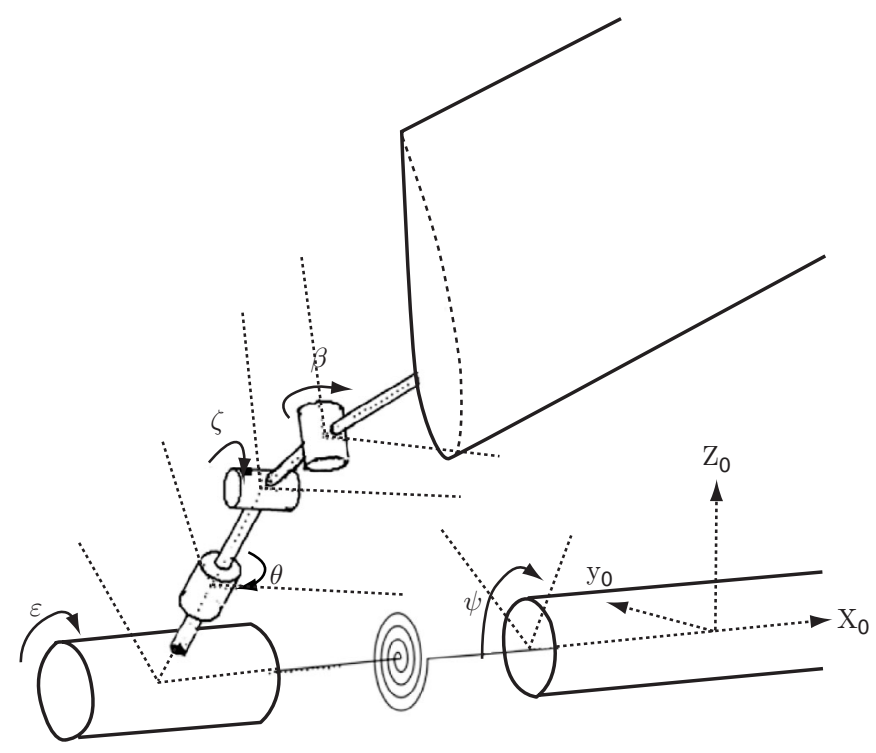

Figure 5: The STABT00L baseline model, a model of an isolated blade. The model has three degrees of freedom: $\varepsilon, \zeta$ and $\beta$. The angle $\theta$ is a constant angle. The shaft rotates with a constant rotational velocity $\Omega$. 
was analysed as a model with three degrees of freedom. However, as only the blade has a mass and inertia, contrary to the description and analysis in [38], it actually contains only two degrees of freedom: the flap and lead-lag angle. The hub rotation is completely dependent on these two variables, therefore the system cannot be described by the three equations given in the paper and the conclusions based on the analytical model are therefore not valid.

The correct equations of motion that govern this system, based on Newton, include the acceleration terms, damping terms and stiffness terms, resulting in the following basic set of equations for small perturbations about the steady state:

$$
\left\{\begin{array}{c}
\tilde{\beta}^{\prime \prime} \\
\tilde{\zeta}^{\prime \prime}
\end{array}\right\}+\left[\begin{array}{cc}
C_{\beta \beta} & C_{\beta \beta} \\
C_{\zeta \beta} & C_{\zeta \zeta}
\end{array}\right]\left[\begin{array}{c}
\tilde{\beta}^{\prime} \\
\tilde{\zeta}^{\prime}
\end{array}\right\}+\frac{1}{I \Omega^{2}}\left[\begin{array}{cc}
k_{\beta \beta} & k_{\beta \zeta} \\
k_{\zeta \beta} & k_{\zeta \zeta}
\end{array}\right]\left[\begin{array}{c}
\tilde{\beta} \\
\tilde{\zeta}
\end{array}\right\}=\left\{\begin{array}{l}
0 \\
0
\end{array}\right\}
$$

Note that the notations used $\widetilde{\beta}$ and $\widetilde{\zeta}$ do not coincide with the $\beta$ and $\zeta$ shown in the figure. $\widetilde{\beta}$ and $\widetilde{\zeta}$ represent small perturbations about the steady state in the directions of $\beta$ and $\zeta$ respectively, but now include a component in the same direction due to the deformation $\varepsilon$ in the hub. The notation for the derivatives used in equation 4 is: ()$^{\prime}=\frac{\partial}{\partial \psi} ;()^{\prime \prime}=\frac{\partial^{2}}{\partial \psi^{2}}$.

To derive the stiffness matrix, assume a moment $M_{\beta}$ acting on the blade about the flap hinge, combined with a moment $M_{\zeta}$ about the lead-lag hinge, which will result in a deformation $\widetilde{\beta}$ :

$$
\tilde{\beta}=\frac{M_{\beta}}{k_{\beta}}+\frac{M_{\zeta} \sin \theta \cos \theta}{k_{\varepsilon}}+\frac{M_{\beta} \sin ^{2} \theta}{k_{\varepsilon}}
$$

and a deformation $\widetilde{\zeta}$ :

$$
\tilde{\zeta}=\frac{M_{\zeta}}{k_{\zeta}}+\frac{M_{\beta} \sin \theta \cos \theta}{k_{\varepsilon}}+\frac{M_{\zeta} \cos ^{2} \theta}{k_{\varepsilon}}
$$

The components in the stiffness matrix in equation 4 can be determined by combining these two equations and rewriting them:

$$
\begin{aligned}
& k_{\beta \beta}=\frac{k_{\beta}\left(k_{\varepsilon}+k_{\zeta} \cos ^{2} \theta\right)}{k_{\varepsilon}+k_{\zeta} \cos ^{2} \theta+k_{\beta} \sin ^{2} \theta} \\
& k_{\beta \zeta}=k_{\zeta \beta}=-\frac{\left(\sin \theta \cos \theta k_{\zeta} k_{\beta}\right)}{k_{\varepsilon}+k_{\zeta} \cos ^{2} \theta+k_{\beta} \sin ^{2} \theta} \\
& k_{\zeta \zeta}=\frac{k_{\zeta}\left(k_{\varepsilon}+k_{\beta} \sin ^{2} \theta\right)}{k_{\varepsilon}+k_{\zeta} \cos ^{2} \theta+k_{\beta} \sin ^{2} \theta}
\end{aligned}
$$

The aerodynamic forces are simplified using a representative section at $\frac{3}{4} R$, assuming that the angle of attack at that point can be used for the entire blade, but taking into account the difference in the local velocity $\Omega r$. The lift- and drag-coefficient are linearised about the steady state condition. With this, the linearised aerodynamic moment can be derived to be:

$$
M_{\beta}=\frac{\gamma}{8} \frac{\Omega^{2} I}{c_{l_{\alpha}}}\left[c_{l_{0}}+2 c_{l_{0}} \tilde{\zeta}^{\prime}-c_{l_{\alpha}} \tilde{\beta}^{\prime}\right]
$$


This gives for the steady state deformation $\widetilde{\beta_{0}}$ :

$$
\tilde{\beta}_{0}\left(1+\frac{k_{\beta 3}}{I \Omega^{2}}\right)=\frac{\gamma}{8 c_{l_{\alpha}}} c_{l_{0}}
$$

and for the moment for small perturbations:

$$
M_{\beta}=\frac{\gamma}{8} \frac{\Omega^{2} I}{c_{l_{\alpha}}}\left[2 c_{l_{0}} \tilde{\zeta}^{\prime}-c_{l_{\alpha}} \tilde{\beta}^{\prime}\right]
$$

Similarly, the equation for the moment for small perturbations in the direction of the leadlag hinge becomes:

$$
M_{\zeta}=\frac{\gamma}{8} \frac{\Omega^{2} I}{c_{l_{\alpha}}}\left[-c_{l_{0}} \tilde{\beta}^{\prime}-2 c_{d_{0}} \tilde{\zeta}^{\prime}+c_{d_{\alpha}} \tilde{\beta}^{\prime}\right]
$$

These moments come back in the equation of motion as damping terms, with the opposite sign.

Next to these aerodynamic terms, the Coriolis forces and the change in centrifugal forces should be taken into account as damping terms, represented by $2 \widetilde{\beta_{0}}$.

Therefore the damping matrix components become:

$$
\begin{aligned}
C_{\beta \beta} & =\frac{\gamma}{8} \quad C_{\beta \zeta}=2 \tilde{\beta}_{0}-\frac{\gamma}{4 c_{l_{\alpha}}} c_{l_{0}} \\
C_{\zeta \beta}=-2 \tilde{\beta}_{0}+\frac{\gamma}{8 c_{l_{\alpha}}}\left(c_{l_{0}}-c_{d_{\alpha}}\right) C_{\zeta \zeta} & =\frac{\gamma}{4 c_{l_{\alpha}}} c_{d_{0}}
\end{aligned}
$$

The aerodynamic terms in the damping matrix correspond to the terms in the matrix calculated in equation 1, if one linearises the terms in the matrix using that the products $V \widetilde{\beta^{\prime}}$ and $V \widetilde{\zeta}^{\prime}$ are small.

The derived equations can be used to determine the damping and frequencies of the system. During the STABTOOL project, the most critical case was concluded to be the socalled 'drag-stall' case, where $c_{l_{0}}-c_{d_{\alpha}}$ approximates zero. The results for the eigenvalues, using the above derived corrected equations, for a case of drag-stall, are plotted in figure 6. Despite the incorrect model being used in the STABTOOL project, the illustrated results correspond well with those obtained during the project [38] and show that it is indeed possible that the flaplag instability will occur for flap eigenfrequencies outside of the $\Omega<\omega_{\beta}<\sqrt{2} \Omega$ range, when operating in or near stall. In the example that has been shown $\omega_{\beta} \approx 4.4 \Omega$ so well outside the aforementioned range.

The damping matrix also illustrates that the instability that was found, is not simply a case of stall induced vibrations as discussed in a previous section, the Coriolis and centrifugal terms in the matrix play an important role in the instability, and if they are not included in the equations, the instability does not show up for the selected values of $\theta$, as illustrated in figure 6 .

However, it must be noted that the most critical case that was assumed in [38] and used to obtain the results illustrated in figure 6 , does not seem to be very realistic. By analysing aerofoil data for representative aerofoils for current day wind turbines it can be shown that 


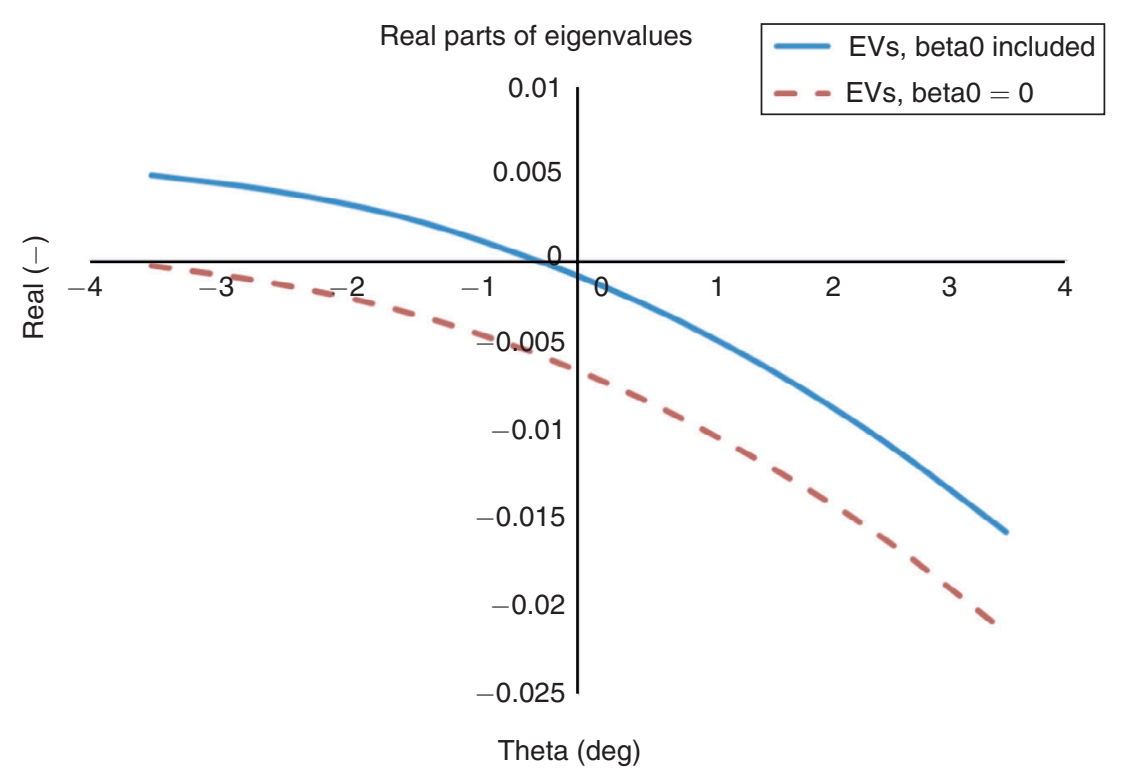

Figure 6: The real parts of the eigenvalues for the equations of motion for different values of the structural pitch angle $\theta$, using $\gamma=13.2, c_{l_{0}}=c_{d_{\alpha}}=1.3, c_{l_{\alpha}}=6.5,0=c_{d_{0}}=0.015, \frac{k_{\varepsilon}}{I \Omega^{2}}=\frac{k_{\zeta}}{I \Omega^{2}}=45.1584$ and $\frac{k_{\beta}}{I \Omega^{2}}=20$. The results using $\beta_{0}=0$ are also shown.

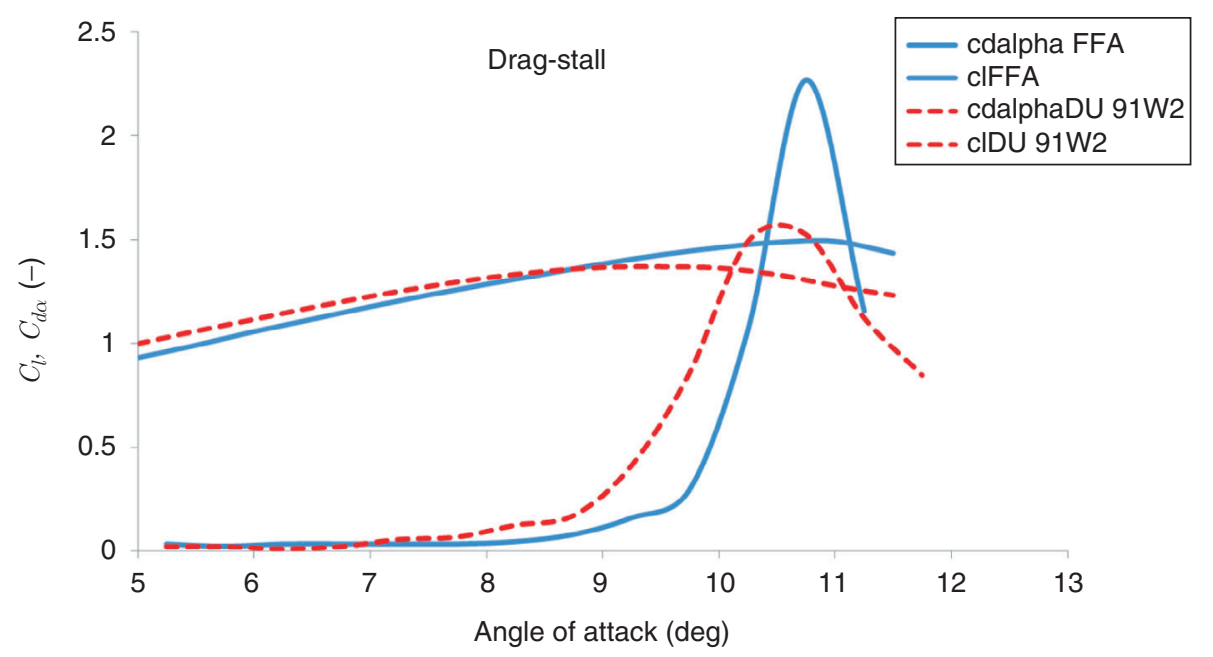

Figure 7: The values for $c_{l}$ and $c_{d_{\alpha}}$ for two different aerofoils, namely FFA-W3-221 at $R e=1.8 \mathrm{M}$ and DU $91 \mathrm{~W} 2-250$ at $2 \mathrm{M}$.

actually the condition of drag-stall $\left(c_{l_{0}}-c_{d_{\alpha}} \leq 0\right)$ will only occur for a handful of aerofoils and for small ranges of angle of attack. This drastically limits the reality of drag-stall occurring in the way that it was assumed in the STABTOOL projects. As an illustration the values for $c_{d \alpha}$ and for the corresponding lift coefficients $c_{l}$ for two of the 'most critical' aerofoils from a selection of typical wind turbine aerofoils are illustrated in figure 7 . The values for $c_{d \alpha}$ were numerically calculated from the drag polars. This figure clearly illustrates the very small range of angles of attack for which the so-called drag-stall condition occurs, if it occurs at all. Next to this, equation 12 shows that the transformation given in equation 2 has been lost due to the 
linearisation of the equations of motion, while in section 2.2 it was shown that the effect of the difference between the in-plane direction and the direction of the first edgewise mode has a significant effect on the damping of this mode.

In [40], the instability that has been observed on the Micon 1500/600 wind turbine, is also attributed to a flap-lag-stall instability, with possible involvement of the tower. However, when looking at the results in this report, the flapwise and edgewise frequency were still very much separated, except for very high wind speeds $(35 \mathrm{~m} / \mathrm{s})$. It seems much more likely that what actually occurred was either the first flap- or first edgewise mode becoming negatively damped or possibly a combination of both. This is similar to the results found in [41], where also both the edgewise and the flapwise mode were negatively damped. What does become clear in [40] is that the limitations on the flap frequency found by Ormiston [33], is no longer valid when structural coupling and stall are included in the models. This is also illustrated in [42] using a semi-qualitative method.

It is difficult to draw any conclusions from these different sources dealing with flap-lag flutter, on whether or not this instability is a future risk for wind turbines. Some instabilities have been found in analytical models, but often for less realistic situations. However, this instability cannot simply be neglected during the design phase. Especially the fact that wind turbines are currently being designed to operate offshore using very different rpms should be taken into account. These higher rpms result in the possibility of the flap frequency actually coming close to the limitations found by Ormiston [33], which would increase the risk of flaplag flutter.

\subsection{Classical flutter and divergence}

Classical flutter and divergence are both known from fixed wing aeroplanes. Of course the centrifugal action on the helicopter or wind turbine blade is not part of the classical flutter of fixed wing aircraft. Due to the centrifugal forces, the flap stiffness is effectively increased.

Classical flutter is a destructive combination of a torsional oscillation with a flapping oscillation. In the case of divergence, it can result in twisting off of the blade. The mechanism can easily be explained. If the blade flaps, the changes in the aerodynamic force act in the aerodynamic centre, but the inertial forces act in the centre of gravity. If the aerodynamic centre is not coinciding with the centre of gravity, the flapping motion will result in a moment about the pitch axis. These moments can be proportional to the acceleration, to the velocity or to the displacement. Therefore there are different phase angles and a destructive interference is possible [10]. Sometimes the term pitch-flap flutter is used, in both helicopter and wind turbine literature, e.g. [10, 22]. In helicopters this instability is prevented by mass balancing of the blades. This is done by adding non-load-carrying mass or ballast to the blades and can be as much as 9-12 percent of the total blade mass [22].

Divergence is only possible when the torsional stiffness is very low, such that the centrifugal twisting moment in flapping is sufficient to drive the blade through stall in a single cycle. For a wind turbine this could be the case when there is a pitch-link failure whereby the stiffness in pitch direction almost completely disappears and divergence could occur. Under the influence of gravity, this could become a limit cycle behaviour.

As pointed out in [11], this instability has so far not occurred on wind turbines. However, it cannot be ruled out for future (larger) designs. As stated by Hansen [12], classical flutter can occur when the flow is attached, the tip speed is high, the torsional stiffness is rather low and the centre of gravity is positioned behind the aerodynamic centre. Both Lobitz [31] and Hansen [12] give extensive descriptions of this possible instability. In these articles it is shown that the 
increase in size to get to the current size of wind turbines has resulted in blades that are closer to the possibility of classical flutter occurring. However, for these blades the flutter speed, above which flutter will occur, is still significantly higher than the operating speeds, but a further increase in size, higher tip speed ratios for offshore or more structural coupling can reduce the difference between operating speeds and flutter speed. Hansen [12] and Lobitz [31] also show that unsteady aerodynamics should be taken into account when analysing the flutter speed, using only quasi steady aerodynamics is a very conservative approach and could lead to designs that are unnecessarily stiff.

The mass balancing of blades as done in helicopters, by adding non-load-carrying mass such that the c.g. is forward of the quarter chord point along the entire blade, would not be realistic for wind turbines. However, the flutter boundary can be significantly extended by changing the position of the c.g. by only a small distance, as shown in [12]. Therefore in cases where the blade is operating too close to the flutter boundary, looking at possibilities of moving the c.g. forward could provide a solution.

\section{FROM BLADE INSTABILITIES TO WIND TURBINE INSTABILITIES}

The discussion above is limited to isolated blades and their possible instabilities. For wind turbines, the tower, shaft, multiple blades and other components also have a significant effect on the natural mode shapes, natural frequencies and corresponding damping coefficients of the complete turbine. For example, in the discussion of negative damping of the edgewise mode of the APX40T blades, it was mentioned that the phase of the three different blades was such, that the drive train could not add any damping to the vibration [7]. This is one illustration of the importance of the multiple blade effects. Another illustration is the difference between the lead-lag frequency and the flap frequency that becomes even smaller if one also takes into account the clamping stiffness as well as the multi-blade effects [17]. Due to the additional hub rotation resulting from the multi-blade effects, the lowest lead-lag frequency will be significantly lower than the frequency that is calculated for an isolated, infinitely stiff clamped blade. Therefore the difference between the flap frequency and lead-lag frequency is reduced, influencing the possibility of the flap-lag instability occurring.

In a complete wind turbine, the isolated blade modes will couple with identical modes on the other blades, and possibly include tower deformations, shaft deformations etc. Forward, backward whirling and collective modes will show up, instead of the modes of the isolated blades, e.g. backward whirling flap mode, symmetrical flap mode and forward whirling flap mode. This results in more complex mode shapes at other frequencies and other possible instabilities and resonances. As was shown for an isolated blade, the mode shape is important for the damping, therefore a change in the mode shape will have a significant effect on the damping of this mode. For example, due to stall, the backward whirling edgewise mode can become negatively damped, which is similar to the possibility of negative damping of the first edgewise blade mode. However the motion of the vibration in the natural mode of the complete turbine is more complex. As analysed by Hansen [43] and measured by Thomsen [44], the backward edgewise whirling mode has less damping than the forward edgewise whirling mode due the the difference in the amount of out-ofplane motion between the two mode shapes, while both concern the same isolated blade mode.

This discussion illustrates that analysis of only a blade is not sufficient to ensure a stable wind turbine, but it does provide important insight to prevent instabilities and knowledge that is needed to alter the design to remove an instability. 


\section{CONCLUSIONS}

Aeroelasticity is an important but complex field for wind energy. There are several dedicated tools available to analyse the aeroelastic stability of a wind turbine. However, knowing the basic possible aeroelastic blade instabilities is crucial in the design of an aeroelastically stable wind turbine. It is important to have a good overview of the possible aeroelastic instabilities and avoid instabilities occurring during normal operation. Especially for blade instabilities, the knowledge obtained over the years within the field of helicopters can be very useful for wind turbine aeroelasticians. However, significant differences between helicopters and wind turbines have also been shown to have a large impact on the stability boundaries. Instabilities that are not known for helicopters have become relevant for wind turbines. Therefore the current knowledge of aeroelastic instabilities for helicopters is not sufficient for wind turbines, the limits that have been found for helicopters cannot be directly applied to wind turbines.

Different blade instabilities have been discussed and situations have been explained in which these could occur. Some examples of stall flutter and flap-lag flutter have been shown. The instability that is currently the most likely to occur on a wind turbine is negative damping of the edgewise mode, which is translated into whirling edgewise modes on a complete turbine. However, other instabilities are shown that cannot be ruled out for current or future (larger) wind turbines. The designs of the wind turbines are still developing in such a way that new instabilities could possibly arise that were not experienced on earlier designs. For example, the offshore turbines will no longer have noise restrictions that limit the tip speed. Therefore the operational conditions of these turbines could be significantly different from the current turbines and instabilities such as flap-lag flutter and classical flutter, should be considered once again.

\section{REFERENCES}

[1] Sullivan, T.L., A Review of Resonance Response in Large, Horizontal-Axis Wind Turbines, in: R. Thresher, ed., Proceedings Wind Turbine Dynamics Workshop, no. CONF - 810226 in NASA Conference Publication 2185, NASA Lewis Research Center, DOE Publication, Cleveland Ohio, 237-244.

[2] Hau, E., Wind Turbines: Fundamentals, Technologies, Application, Economics, 2nd edn., Springer-Verlag, Berlin Heidelberg, 2006.

[3] Pavel, M.D., An Investigation of the Rotor - Tower Instability of the KEWT Wind Turbine, Tech. Rep. Memorandum M-879, Faculty of Aerospace Engineering, Delft, 1999.

[4] van Holten, T., Analysis of the Vibrations of the KEWT Wind Turbine, Tech. Rep. intern report, SPE, Amsterdam, 1987, in Dutch.

[5] Stiesdal, H., Extreme Wind Loads on Stall Regulated Wind Turbines, in: BWEA 16, Mechanical Engineering Publications Ltd., Stirling, UK.

[6] Møller, T., Blade cracks signal new stress problem, WindPower Monthly, May.

[7] Anderson, C., Heerkes, H. and Yemm, R., The use of blade-mounted dampers to eliminate edgewise stall vibration, in: EWEA 1999, Nice, France.

[8] Friedmann, P.P., Aeroelastic stability and response analysis of large horizontal-axis wind turbines, Journal of Wind Engineering and Industrial Aerodynamics, 5(3-4), (1980), 373 - 401, doi:10.1016/0167-6105(80)90043-4, wind Energy Conversion Systems.

[9] Rasmussen, F., Hansen, M.H., Thomsen, K., Larsen, T.J., Bertagnolio, F., Johansen, J., Madsen, H.A., Bak, C. and Hansen, A.M., Present Status of Aeroelasticity of Wind Turbines, Wind Energy, 6, (2003), 213-228. 
[10] Eggleston, D.M. and Stoddard, F.S., Wind Turbine Engineering Design, Van Nostrand Reinhold Company, New York, USA, 1987.

[11] Pavel, M.D. and Schoones, M.M.J., Literature Survey on Aeromechanical Instabilities for Helicopters and Wind Turbines, Tech. Rep. Memorandum M-877, Faculty of Aerospace Engineering, Delft, 1999.

[12] Hansen, M.H., Aeroelastic Instability Problems for Wind Turbines, Wind Energy, 10, (2007), 551-577.

[13] Hansen, M. O. L. et al, State of the art in wind turbine aerodynamics and aeroelasticity, Progress in Aerospace Sciences, 42, (2006), 285-330.

[14] Petersen, J.T. and et al., Prediction of Dynamic Loads and Induced Vibrations in Stall, Tech. Rep. Technical Report Risø-R-1045(EN), Risø National Laboratory, Roskilde, Denmark, 1998.

[15] Rasmussen, F., Petersen, J.T. and Madsen, H.A., Dynamic Stall and Aerodynamic Damping, Journal of Solar Energy Engineering, 121(3), (1999), 150-155, doi:10.1115/1.2888426.

[16] Hansen, M.H. and Buhl, T., Design guidelines for passive instability suppression-Task-11 Report, Tech. Rep. Risø-R-1575(EN), RisøNational Laboratory, Roskilde, Denmark, 2006.

[17] Holierhoek, J.G., Aeroelasticity of Large Wind Turbines, Ph.D. thesis, Delft University of Technology, 2008.

[18] Holierhoek, J.G., Investigation into the possibility of flap-lag-stall flutter, in: 45th AIAA Aerospace Sciences Meeting and Exhibit, Reno.

[19] Lundsager, P., Petersen, H. and Frandsen, S., The Dynamic Behavior of theStallRegulated Nibe A Wind Turbine Measurements and a Model for Stall-Induced Vibrations, Tech. Rep. Technical Report Risø-M-2253, Risø National Laboratory, Roskilde, Denmark, 1981.

[20] Dekker, J.W.M., de Groot, C.M. and Spath, M., Mechanical Measurements on VSH 20WPX-THR Rotor Blades at the '25 HAWT' Rotor Test Facility-Part 1, Tech. Rep. ECN-8935, ECN, Petten, the Netherlands, 1989.

[21] Chou, P.C., Pitch-Lag Instability of Helicopter Rotors, Journal of the American Helicopter Society, 3(3), (1958), 30-39, doi:10.4050/JAHS.3.30.

[22] Bramwell, A.R.S., Done, G. and Balmford, D., Bramwell's Helicopter Dynamics, 2nd edn., Butterworth-Heinemann, Oxford, 2001.

[23] Kallesøe, B.S. and Hansen, M.H., Effects of Large Bending Deflections on Blade Flutter Limits, UpWind Deliverable D2.3, Tech. Rep. Technical Report Risø-R-1642(EN), Risø National Laboratory, Roskilde, Denmark, 2008.

[24] Kallesøe, B.S., Effect of steady deflections on the aeroelastic stability of a turbine blade, Wind Energy, 14, (2011), 209-224, doi:10.1002/we.413.

[25] Johnson, W., Helicopter Theory, Princeton University Press, 1980.

[26] Loewy, R.G., Review of Rotary-Wing V/STOL Dynamic and Aeroelastic Problems, Journal of the American Helicopter Society, 14(3), (1969), 3-23, doi: 10.4050/JAHS.14.3.

[27] Dekker, J.W.M., TEST FLEXTEETER, Tech. Rep. ECN-C-93-072, ECN, Pet-ten, the Netherlands, 1993.

[28] van Kuik, G. and Dekker, J., The FLEXHAT program, technology development and testing of flexible rotor systems with fast passive pitch control, Journal of Wind 
Engineering and Industrial Aerodynamics, 39(1-3), (1992), 435 - 448, doi:DOI: 10.1016/0167-6105(92)90567-T.

[29] Dowell (editor), E.H., Curtis Jr., H.C., Scanlan, R.H. and Sisto, F., A Modern Course in Aeroelasticity, Third Revised and Enlarged Edition, Kluwer Academic Publishers, Dordrecht, 1995.

[30] Katz, J. and Plotkin, A., Low Speed Aerodynamics - From Wing Theory to Panel Methods, McGraw-Hill, New York, 1991.

[31] Lobitz, D.W., Aeroelastic Stability Predictions for a MW-sized Blade, wind energy, 7, (2004), 211-224.

[32] Ormiston, R.A., Investigations of Hingeless Rotor Stability, Vertica, 7:2, (1983), 143-181.

[33] Ormiston, R.A. and Hodges, D.H., Linear Flap-Lag Dynamics of Hingeless Rotor Blades in Hover, Journal of the American Helicopter Society, 17:2, (1972), 2-14.

[34] Huyer, S.A., Simms, D.A. and Robinson, M.C., Unsteady Aerodynamics Associated with a Horizontal-axis Wind Turbine, AIAA Journal, 34, (1996), 1410-1419.

[35] Ormiston, R.A. and Bousman, W.G., A Study of Stall-Induced Flap-Lag Instability of Hingeless Rotors, in: Presented at the 29th Annual National Forum of the American Helicopter Society, American Helicopter Society, Washington D.C.

[36] van Holten, T., Pavel, M.D. and Smits, G.N., Aeroelastic Stability of Modern Windturbines, Final Report Phase 1, Tech. Rep. Memorandum M-880, Faculty of Aerospace Engineering, Delft, 1999.

[37] van Holten, T., Final report STABTOOL phase II, Aeroelastic Tools to asses the stability of large wind turbines, Tech. Rep. FM\&P00.016, Faculty of Aerospace Engineering, Delft, 2000.

[38] Holierhoek, J.G., van Holten, T. and Mulder, T.J., Automatic Simulation to Determine the Aeroelastic Stability of Large Scale Wind Turbine Rotors (With Emphasis on Flap-LagStall Flutter), in: 26th European Rotorcraft Forum in conjunction with 15th European Helicopter Association Symposium Proceedings, 69, The Hague, the Netherlands.

[39] Thakoer, R., van Kuik, G.A.M. and van Leeuwen, H.L., Classification of future 5 MW turbines, by extrapolation of current trends, Tech. Rep. Ivw 99160R, Faculty of Civil Engineering and Geosciences, Delft, 1999.

[40] Pavel, M.D., An investigation of the Lead-Lag Instability of the Micon 1500/600 Wind Turbine, Tech. Rep. Memorandum M-878, Faculty of Aerospace Engineering, Delft, 1999.

[41] Chaviaropoulos, P.K., Flap/Lead-Lag Aeroelastic Stability of Wind Turbine Blade Sections, Wind Energy, 2, (1999), 99-112.

[42] van Holten, T., Energy flow considerations, aneducational tool to clarify aeroelastic phenomena, in: 26th European Rotorcraft Forum in conjunction with 15th European Helicopter Association Symposium Proceedings, 66, The Hague, the Netherlands.

[43] Hansen, M.H., Improved Modal Dynamics of Wind Turbines to Avoid Stall-induced Vibrations, Wind Energy, 6, (2003), 179-195.

[44] Thomsen, K., Petersen, J.T., Nim, E., Øye, S. and Petersen, B., A Method for Determination of Damping for Edgewise Blade Vibrations, Wind Energy, 3, (2000), 233-246. 